\title{
El uso de los trastornos de la personalidad para la delimitación de los personajes femeninos con problemas de sociabilización en la ficción norteamericana actual.
}

Laura Pacheco Jiménez

Universidad de Sevilla http://dx.doi.org/10.12795/AdMIRA.2018.01.02

\section{RESUMEN}

Los personajes con problemas para establecer relaciones sociales con otros son un recurso muy utilizado en la ficción norteamericana actual, desde House (Fox, 2004-2012) a Eliot Anderson (Mr. Robot, USA Network, 2015), pasando por series como Bones (Fox, 2005-2017), El mentalista (2008-2015) o Homeland (Showtime, Flox21, 2011) entre otras.

La proliferación de seres que no saben, no pueden o no quieren mantener relaciones personales viene determinada por un trauma previo que provoca en el personaje un nihilismo inherente a la creación del yo que lo hace único dentro de su universo. Sin embargo, tras el análisis de una trentena de series de televisión norteamericanas emitidas entre los años 2005-2016, se ha establecido que hay una tendencia a delimitar a los personajes femeninos con un trastorno de la personalidad en mayor medida que a los personajes masculinos, que gozan de una ambigüedad que los hace más interesantes al espectador.

\section{PALABRAS CLAVE}

Series de televisión, trastornos de la personalidad, personajes asociales, televisión y género, relaciones sociales.

\begin{abstract}
The characters with problems to establish social relationships with others are a resource widely used in American fiction, from House (Fox, 2004-2012) to Eliot Anderson (Mr. Robot, USA Network, 2015), through series such as Bones (Fox, 2005-2017), The Mentalist (2008-2015) or Homeland (Showtime, Flox21, 2011) among others.
\end{abstract}


The spread of characters who do not know, can not or do not want to maintain personal relationships is determined by a previous trauma that provokes in the character a nihilism inherent to the creation of the self that makes him unique within his universe. However, after the analysis of some thirty American television series issued between 2005-2016, it has been established that there is a tendency to delimit female characters with a personality disorder to a greater extent than male characters, that enjoy an ambiguity that makes them more interesting to the viewer.

\section{KEYWORDS}

TV series, personality disorders, asocial characters, TV and gender, social relationships. 
"Las culturas pueden distinguirse unas de otras por los héroes a quiénes más admiran. En muchos casos el héroe tiene un estilo bien definido del comportamiento social".

(Argyle, 1978:136)

\section{Introducción}

Que el héroe tiene una función social y, a la vez, supone un modelo de comportamiento para el ser humano es algo completamente innegable desde el siglo pasado, así lo demuestra Argyle, uno de los grandes exponentes de la psicología social del siglo XX. La necesidad imperante y mesiánica del ser humano de construir personajes a su imagen y semejanza no es más que otra demostración del complejo de Dios que le invade a la hora de crear. Es por esto que cada relato audiovisual, personaje e incluso técnica ha de entenderse y estudiarse en el contexto en el que se produce para llegar a comprender su significación completa.

Desde finales de los años noventa hasta ahora, uno de los temas que más ha interesado a la ficción serial han sido los asesinatos. No obstante, el punto de vista de la investigación del crimen ha ido variando desde el agente Cooper (Twin Peaks, ABC, 1990-1991), hasta Dexter Morgan (Dexter, Showtime, 2006-2013), el asesino en serie más querido de la historia de la televisión. Series como Mentes Criminales (CBS, 2005-2016), El mentalista (CBS, 2008-2015) o Hannibal (NBC, 2013-2015) han hecho de la representación del asesinato un juego estético con el que atrapar al espectador y de la resolución del crimen un paradigma narrativo que ha cautivado al público temporada tras temporada. En cambio, al margen de los asesinatos, hay algo que estas y otras series tienen en común: la inadaptación social de alguno de sus personajes, y no exclusivamente de los asesinos. Estos asociales, lo son generalmente por un trauma anterior al punto de partida de la ficción. Es el caso del doctor Spencer Reid (Mentes Criminales), que tiene un cociente intelectual muy por encima de la media, memoria eidética, pero problemas sociales derivados de haber pasado gran parte de su adolescencia cuidando a su madre esquizofrénica, después de que su padre los abandonara. Patrick Jane (El mentalista), utilizaba su virtud de observar y deducir, para un programa televisivo, aunque decide ponerlo al servicio de la policía cuando su mujer y su hija son asesinadas impunemente. Y, por último, Will Graham (Hannibal), personaje emocionalmente inestable y sin habilidades sociales cuyo don y castigo es el exceso de empatía, lo que le permite meterse en la piel del asesino 
trabajando con la policía para resolver los casos. Estos son apenas algunos ejemplos de cómo, a partir de las series de crímenes, surgen personajes con escasas habilidades sociales, con un trauma personal y con una necesidad de suplir sus carencias a la hora de relacionarse aportando un beneficio a la comunidad desde su puesto de trabajo.

La presente investigación tiene como punto de partida un estudio previo realizado entre 20152016 titulado Héroes asociales en la ficción televisiva estadounidense actual: patologías clínicas y psicoanalíticas de los personajes de 'House' a 'Mr. Robot' (Pacheco, 2015, inédita), en el que quedaron analizados y retratados un total de treinta personajes procedentes de veinte series de televisión - no todas norteamericanas puesto que se establecen semejanzas entre la ficción serial estadounidense y otros casos interesantes procedentes de otros países-. Las premisas fundamentales eran dos: que los personajes tuvieran problemas de relaciones sociales y que, los procedentes de series de asesinatos, no fueran el asesino. Los únicos casos que se salieron de las premisas base fueron el ya mencionado Dexter Morgan y Light Yagami (Death Note, DN Dreams, 2006-2007), cuya comparación tanto entre sí como con el resto de personajes del estudio sirvió para determinar aspectos esenciales de los trastornos de personalidad, especialmente el bipolar, y complejo de Dios, así como para analizar la importante presencia de este tipo de antihéroes $-\mathrm{O}$ villanos e, incluso antivillanos- dado que ambas series aparecieron el mismo año en dos puntos muy alejados del mundo y las semejanzas entre ambas son más que notables.

El pasado estudio pretendía llegar a un retrato generalizado de este tipo de personajes y de su función social por lo que, en primera instancia, las series fueron escogidas de 2005 a 2015 por su relevancia y conocimiento por parte del público, hecho que resultó muy revelador y cuyas deducciones han originado la presente investigación. De la veintena de ficciones analizadas, solo ocho de ellas estaban protagonizadas por mujeres -aquí, además, hay que tener en cuenta que Los Misterios de Laura fue incluida tanto la versión española (TVE, 2009-2014), como la adaptación estadounidense (NBC, 2014), por lo que es el mismo caso contabilizado dos veces-. Además, de los treinta personajes, sólo seis son protagonistas absolutas femeninas y solo hay un caso -Castle- de coprotagonismo hombre-mujer, que, aunque el peso de los personajes es compartido, el título que da nombre a la ficción es el apellido del protagonista masculino quien, por cierto, es el autor de renombre y ella hace las veces de musa. Sin embargo, al analizar a 
personajes, no todo es cantidad, sino que la calidad importa. A rasgos generales, aquellas series en las que el protagonista era masculino gozaban de mayor relevancia social (House, Dexter, Hannibal, True Dectective, Mr. Robot) y sus personajes eran más interesantes. A la hora de la construcción, los personajes masculinos tenían un background más elaborado que los femeninos; mientras que eran más ambiguos, lo que les da, además, un halo de misterio que resulta apasionante para el espectador. Ellas están más definidas psicológicamente (Bones, Homeland, Girls) pero, salvo excepciones como Orphan Black, son sujetos cuyo pasado es apenas una excusa y no tiene la misma importancia para el presente narrativo de la ficción como ocurría en los casos de ellos. Un ejemplo es Mr. Robot, cuyo protagonista sufre paranoia esquizoide y es su enfermedad lo que construye todo el discurso de la primera temporada. Por el contrario, en series como Homeland, donde Carrie Mathison sufre bipolaridad, su enfermedad no es más que aquello que le da credibilidad o no de cara al resto de personajes, la delimita en sus acciones, pero no la define en su arco narrativo, lo que la hace evolucionar es su obstinación -como puede ocurrir con cualquier personaje de una serie de similares características-. En definitiva, esto no son más que algunos ejemplos que ponen de relieve la necesidad de un estudio tanto de los personajes asociales y su importancia en la sociedad actual, como la importancia de incluir a féminas con la misma calidad en la construcción, mujeres cuya necesidad fundamental no sea reivindicar su posición, sino ocuparla para ser asociales, abstractas, ególatras y todos aquellos adjetivos que actualmente son atribuibles a los personajes masculinos puesto que, en el caso de ellos, no se tienen por qué definir por su género, sino por el problema que los separa de ser héroes clásicos y los han convertido en antihéroes, villanos o antivillanos dando origen al momento de más interés psicológico y sociológico de la historia de la televisión.

Debido a esto, la presente investigación pretende servir de modelo definitorio de aquellos personajes femeninos cuyos problemas psicológicos delimitan su forma de interactuar con su contexto. Así pues, se efectuará un acercamiento a la psicología y a las formas que el ser humano tiene en su haber para desarrollar habilidades sociales y, a su vez, se definirán a estos personajes desde el punto de vista narrativo, dándole una concordancia dentro de su propia serie para, posteriormente, extrapolarlo y analizar su función social como personajes en un contexto complicado, donde las relaciones se deterioran, la comunicación interpersonal se 
relega y los problemas psicológicos siguen siendo un tabú. Si, como dijo Michael Argyle, el comportamiento del héroe tiende a tener una base bien definida del comportamiento social, ¿por qué no tenemos suficientes heroínas de calidad en el panorama televisivo actual?

\section{Metodología}

La metodología ha sido combinada entre metodología de campo y estudio intensivo de casos (Arias, 1997:31).

En primer lugar, se ha realizado una búsqueda de series con mayor relevancia social de los últimos años con personajes asociales femeninos como protagonistas. Los resultados obtenidos han sido que las series que cumplen estos requisitos son: Bones, Homeland y Orphan Black. Al considerar que tres sujetos no son suficientes para la presente investigación, se ha procedido a una segunda búsqueda más exhaustiva sin tener en cuenta el hecho de la aceptación del público y se han encontrado las series: United States of Tara, Girls, The Bridge, Unreal y Lady Dynamite.

Una vez estudiados los personajes se ha llegado a la conclusión que los problemas de sus dificultades para las relaciones sociales provienen de traumas pasados y que, por lo general, con el tiempo han desarrollado en su mayoría algún tipo de trastorno de la personalidad. Así pues, el marco teórico ha sido elaborado por conceptos propios de la psicología, basados en los trastornos mentales, por lo que la bibliografía de referencia ha sido el DSM-IV TR Manual diagnóstico y estadístico de los trastornos de la personalidad.

Dado que el panorama de personajes que cumplen estos requisitos es reducido se ha aplicado el marco teórico de la psicología, así se ha conseguido el objetivo del estudio intensivo de casos, “este diseño indaga de manera exhaustiva, buscando la máxima profundidad” (Arias, 1997:33) que, en esta investigación servirá para la elaboración de un análisis que permita esbozar las aristas más complejas de la psicología de los personajes que componen el corpus.

\section{Desarrollo teórico o discusión}

\subsection{Enfermedad mental y género, ¿existe sesgo en el diagnóstico?}

Es habitual que, en los manuales de diagnóstico, aparezca un apartado o nota aclaratoria hacia el final donde se establecen proporciones de si la enfermedad o trastorno en cuestión afecta 
mayoritariamente a hombres o a mujeres. Y, también es habitual, que se tiendan a asociar ciertas etiquetas según a que sexo. Theodore Millon dice al respecto que enfermedades como el TNP -Narcisista de la personalidad- se diagnostica con criterios que "habitualmente se asocian a un estilo de vida masculino saludable como tener un exagerado sentimiento de la propia importancia, una preocupación por fantasías de éxito y poder ilimitados, el sentimiento de tener derecho sobre los demás, la falta de empatía y una actitud arrogante"; sin embargo, trastornos como el de la personalidad por dependencia o el histriónico, se consideraron femeninos en tanto que ambos se basan en la inestabilidad y dependencia emocional (Millon et al, 2006:8587). Si bien es cierto que hay enfermedades que, sea cual fuere el motivo, afectan a un sexo u a otro según cuestiones biológicas, cada vez son más los expertos que se muestran cautelosos a la hora de afirmar que un trastorno de la personalidad afecta más a mujeres o a hombres. Véase el caso del DSM-IV-TR, el manual de cabecera que acuna esta investigación, que, en su apartado sobre los diagnósticos por sexos, añade oraciones abiertas, sugerencias, pero no sentencias, como, por ejemplo:

"los estudios epidemiológicos sugieren que la fobia social es más frecuente en las mujeres; sin embargo, cuando se estudian poblaciones clínicas, ambos sexos se muestran igualmente propensos, o bien se constata que el trastorno incide más frecuentemente en los hombres" (2002:505).

O, por poner otro ejemplo:

"Ha habido un cierto interés en considerar si el trastorno antisocial de la personalidad podría infradiagnosticarse en mujeres, sobre todo por el hecho de que en la definición del trastorno disocial se insiste de manera especial en los items de agresividad" (2002:787).

No obstante, el citado manual no es el único ejemplo de esta práctica cada vez más extendida, sino que, por ejemplo, los autores Martos, Ayuda, González, Freire y Llorente, en El síndrome de asperger. Evaluación y tratamiento, han tenido a bien incluir un capítulo sobre las diferentes controversias que genera esta enfermedad aún tan desconocida y, entre ellas, destaca el apartado ¿Dónde están las mujeres con síndrome de Asperger? (Martos et al., 2012:41) del que 
a continuación se hace una síntesis para conocer, en esta enfermedad concreta, qué ocurre con el sesgo de género en el diagnóstico.

Diferentes investigaciones se han centrado a lo largo de las últimas décadas en delimitar qué porcentaje de mujeres padecen SA, sin embargo, pocas han llegado a establecer un conceso. En los años 80 , se estimó que, del grueso de pacientes, habría una mujer por cada diez hombres padeciendo el trastorno, a principios de los 90, que alrededor de una por cada cuatro; pero ninguna de las aproximaciones ha coincidido con las predicciones de los estudios epidemiológicos, por lo que, al menos sobre el papel, las pruebas indican que se está produciendo una infradiagnosticación del SA en las mujeres.

Este teórico error en el proceso de diagnóstico del trastorno tiene consecuencias devastadoras para mujeres que, al no ser etiquetadas clínicamente como asperger, no hallan justificación plausible a sus comportamientos de los que, en la mayoría de las ocasiones, son conscientes de que no son normotípicos.

Desde la infancia, el diagnóstico -o ausencia de él- ya radica en diferencias de ente lo que cabe esperar de una niña o lo que se espera de un varón. Esto se debe a que hay una tendencia en las féminas con SA a tender a la inhibición mientras que los niños tienden a mostrar conductas agresivas, lo que provoca que su sintomatología pase desapercibida con mayor frecuencia para los progenitores. Cuando las niñas establecen sus primeras interacciones se espera de ellas una conducta prosocial y maternal y, en tanto que es lo esperado y transmitido, aquellas que se identifican con las normas aprendidas, la llevan a cabo. Debido a esta conducta comunitaria, aquellas que padecen SA intuyen formas de comportamientos de su grupo de iguales lo que hace, de nuevo, más difícil el diagnóstico, pero, al fin y al cabo, trae ventajas más provechosas para los primeros años de las pacientes.

Una vez llegada la adolescencia, la situación se invierte y son ellas las que gozan de menores índices de apoyo y comprensión, por lo que es una etapa especialmente difícil cuando, además, no han llegado a ser diagnosticadas.

A lo largo de la vida de una mujer, una de esas enseñanzas sociales -aunque tiene una base biológica predominante- es a mostrar empatía, esta presión social de preocupación hacia el otro 
en féminas hace que, a pesar de que ellos sean diagnosticados en mayor número, ellas necesitan de más ayuda psicológica cuando llegan a la edad adulta. Las deficiencias en las relaciones sociales y la falta de satisfacción en sus prácticas hacen que estas mujeres se vuelquen en sus carreras laborales, lo que da unos índices elevados de éxito profesional en pacientes con SA.

Visto el caso concreto expuesto por Martos et al, quedan patente los entresijos que se esconden tras el problema. Un sesgo de estas características puede ocurrir en diferentes partes del proceso, bien desde los criterios diagnósticos del DSM-IV-TR aunque, como se ha demostrado, hay una preocupación por mostrar cierta apertura; bien por los médicos, que diagnostican en función de prejuicios -expuestos en diferentes manuales, lo que tampoco ayuda a solventar el problema-, o incluso desde las muestras para investigaciones en los trastornos de la personalidad cuyos pacientes están ilustrados con los mismos parámetros expuestos. Todo ello conduce al mismo lugar, a una cultura misógina donde el campo de la medicina no está libre de culpa, en palabras de Theodore Millon, "hemos realizado una tarea que deja bastante que desear a la hora de buscar muestras representativas para realizar estadísticas fiables de prevalencia" (2006:85).

\subsection{Trastornadas en series: ¿existe sesgo en la representación?}

Encontrar bibliografía sobre medicina y psicología aplicada a las series de televisión tiende a reducir el campo a artículos científicos recientes, interesantes y rigurosos, pero que demuestran que, a pesar de la popularidad surgida alrededor de este tipo de ficciones, la investigación aún tiene mucho recorrido por delante. Algunos manuales, como Patologías de la verdad (Vargas, 2012), se concentran en una única serie, en este caso House, M.D.; otros autores optan por abrir sus horizontes y realizar una aproximación general a la descripción del panorama, y he aquí donde se encuentran resultados que interesan a nivel de representación. Porque, ¿cómo se eligen las ficciones a analizar en un manual cuya intención es describir el panorama general? Se entiende que los criterios son, bien por popularidad, bien por criterios de los autores centrados en series que, a su juicio, son de calidad. Ya lo advierte Toni de la Torre en su Historia de las series, la mayor compilación de análisis de ficciones televisivas que se haya abarcado en nuestro idioma: 
“cualquier ensayo histórico responde, inevitablemente, a la elección subjetiva del autor que debe escoger aquellos aspectos que considere más relevantes, construyendo un relato propio sobre la evolución de la historia. Así pues, este libro incluye todas aquellas series y datos que el autor ha considerado indispensables e interesantes para explicar la historia de las series de televisión” (De la Torre, 2016:9-10).

La presente consideración no es, ni mucho menos, una crítica personalizada al autor, en tanto que ha realizado un impecable y riguroso trabajo documental; sin embargo, no puede escaparse, que en un manual donde se citan más de un millar de series, hay notables ausencias -es de insistir que no es posible la citación de todas las series que hayan existido-. En el presente estudio se han buscado ex profeso series cuyos personajes protagonistas fueran féminas con problemas de sociabilización. A pesar de que son unos ítems bastante concretos, en la lista de las más de mil series de De la Torre faltan -de las que conforman el presente corpus-:

- Bones.

- United States of Tara.

- Orphan Black.

- Unreal.

- Lady Dynamite.

De estas 6, hay que señalar que Unreal y Lady Dynamite no se encontraban en emisión en el momento de la publicación del libro. Por lo tanto, las que verdaderamente no han sido consideradas en el compendio son Bones, United States of Tara y Orphan Black. Al al menos el primer y el último caso resultan llamativos.

Por poner otro ejemplo, el mismo autor coordina en el año 2016, un libro titulado Medicina y las series de televisión en el que se analiza la influencia de la medicina en series de ficción de relevancia. El libro consta de 16 capítulos, uno de ellos introductorio; de los 15 que restan solo hay dos en los que se analizan series que incluyen a una alguna protagonista femenina entre varias ficciones. El primero, Angels in America, The Normal Hearth y Positius (Clotet y Clotet, 2016:73) -la primera coprotagonizada por hombre-mujer, la segunda por dos hombres y la tercera por dos mujeres-. En el siguiente capítulo se examinan en conjunto Nip/Tuck - 
protagonizada por dos médicos varones- y Anatomía de Grey y la cirugía plástica (Vaquero, 2016:79).

Además, hay otros dos apartados que sí se dedican exclusivamente a una serie protagonizada por un personaje femenino cada uno y los títulos son llamativos:

- Homeland y el mundo de las emociones (Vehil y Lalucat, 2016:105).

- Olive Kitteridge y la depresión ${ }^{1}$ (Estrada, 2016:111).

Obsérvese que desde el nombre de los capítulos se asocian valores tradicionalmente femeninos, al contrario que otros titulados House y el diagnóstico médico (Sanders, 2016:13), Los Soprano y el psicoanálisis (Estrada, 2016:31) o CSI y la medicina forense (Farré, Torrens, Baños y Farré, 2016:99) -de nuevo aquí sorprende la ausencia de Bones, por ejemplo-.

Lejos de intentar cuestionar el sentido crítico de los autores, lo que se pretende es arrojar luz sobre la necesidad de que, en los escasos manuales específicos que existen sobre el tema, se incluyan a personajes femeninos puesto que existir, existen. Además, se tiene en consideración que no se han incluido determinadas series de televisión porque no cuentan con la popularidad suficiente, como es el caso de United States of Tara, lo que sirve de ejemplo para aclarar que el tándem series de televisión, mujeres protagonistas y problemas de sociabilización, no tiene la misma repercusión que si, en la ecuación, se suprime el término "mujeres" y se sustituye por el vocablo "hombres"2.

Al margen de este apunte sobre la representación de estos personajes en libros publicados y con buena aceptación por críticos e investigadores de diversos ámbitos, hay conceptos de otras ramas muy relevantes para la concepción de personajes que copan la pequeña pantalla actualmente y no son exclusivamente de un género o de otro, ni siquiera asociales o no, son

\footnotetext{
${ }^{1}$ Olive Kitteridge no ha sido incluida en el presente estudio en tanto que es una miniserie de 4 capítulos y los sujetos de estudio se han tenido en cuenta por su continuidad en el tiempo o su intención de permanecer en emisión a pesar de contar con temporada única hasta la fecha.

${ }^{2}$ Es interesante pararse a ver que, tal y como se mencionó en el apartado referente al sesgo en el diagnóstico, cuestiones de construcción social como, por ejemplo, "características que una mujer debe tener" provocan una infradiagnosticación en determinadas enfermedades mentales porque se asume que el género al completo tiene ciertos rasgos comunes. Sin embargo, al trasladarse a la pequeña pantalla, son las mujeres las enfermas o trastornadas, siendo una tarea harto compleja hallar a personajes masculinos con trastornos de la personalidad diagnosticados -una excepción a esta regla es Eliot Anderson en Mr. Robot-.
} 
rasgos que identifican a la sociedad actual y que se traspasan a las series de televisión constituyendo un espejo sobre el que el espectador puede deleitarse con una imagen distorsionada -y, normalmente- mejorada de sí mismo. En palabras de Guarinos, "las teorías psicoanalíticas aplicadas a teatro descubren personajes que se desconocen a sí mismos y que conforme se desarrollan se van conociendo, con teorías de Freud y Jung” (2008:6). Así, aplicándolo al panorama televisivo en el contexto actual de la hibridación, los personajes se muestran descubriéndose a sí mismos a la vez que el espectador, activo, puede conocer y reflexionar sobre partes de su ser que no había concebido con anterioridad y por este motivo, los contenidos audiovisuales son medios de comunicación de masas con un potencial sin precedentes.

\subsection{Breve descripción de los TP que afectan a los personajes}

En primer lugar, abordar la definición de trastorno de la personalidad es una tarea harto complicada incluso para especialistas en medicina dado que esta definición trae consigo históricamente una indefinición implícita. El DSM-IV-TR reconoce estas limitaciones y las atribuye a un concepto arcaico pero que aún perdura en manuales de psicología clínica, que no es otro que el hecho de que el término «trastorno mental» implica una distinción entre trastornos «mentales» $\mathrm{y}$ «físicos» cuando de hecho, está demostrado que ambos conceptos están relacionados y que problemas mentales tienen que ver con cuestiones físicas, y viceversa. Aun así, la definición que aporta en sus páginas el manual es la siguiente:

"síndrome o patrón comportamental o psicológico de significación clínica, que aparece asociado a un malestar (p. ej., dolor), a una discapacidad (p.ej., deterioro en una o más áreas de funcionamiento) o a un riesgo significativamente aumentado de morir o de sufrir dolor, discapacidad o pérdida de libertad. Además, este síndrome o patrón no debe ser meramente una respuesta culturalmente aceptada a un acontecimiento particular (p.ej., la muerte de un ser querido). Cualquiera que sea su causa, debe considerarse como la manifestación individual de una disfunción comportamental, psicológica o biológica. Ni el comportamiento desviado (p.ej., político, religioso o sexual) ni los conflictos entre el individuo y la sociedad son trastornos mentales, a no ser que la desviación o el conflicto sean síntomas de una disfunción” (2002:XXIX). 
Uno de los grandes retos tanto de la psicología como de la medicina, es la historia evolutiva de los trastornos de la personalidad, ¿cómo se desarrollan? ¿Qué factores son determinantes? ¿Prima la biología? ¿El contexto social? ¿Un grave trauma es suficiente para desarrollar un trastorno de la personalidad? El problema al que se enfrenta la patogenia durante el desarrollo, tal y como se le llama a este campo en la psicología, o la etiología, como se le denomina en medicina, es que se trata de un cúmulo de factores. Los trastornos de la personalidad se caracterizan por su condición genética, ambiental, traumática y familiar, lo que hace de esta una ardua tarea que llevar a cabo por psicólogos, psiquiatras y médicos de otras ramas, así como investigadores.

Así pues, la presente investigación se acoge a las definiciones ofrecidas por el mencionado manual -aun a sabiendas de las reconocidas deficiencias de este- dado que, a día de hoy, es el manual más completo que existe en el campo de los trastornos de la personalidad. A partir de las definiciones encontradas en sus páginas se detalla a continuación una relación alfabética de los trastornos que afectan a los personajes de la presente investigación de manera resumida.

\subsubsection{Trastorno Asperger (SA)}

El Trastorno de Asperger o Síndrome de Asperger (SA), se define como una "incapacidad grave y permanente para la interacción social y la presencia de pautas de conducta, intereses y actividades repetitivos y restringidos" (DSM-IV-TR, 2002:91).

Sin haber retrasos mentales ni en el aprendizaje del lenguaje, el SA afecta a aspectos más sutiles de la comunicación social habitual. En casos en adultos, el individuo puede mostrar interés por la amistad, pero no conocer los códigos y convenciones de interacciones sociales, por lo que es posible que no compartan con sus amistades intereses o sentimientos, lo que tiende a desembocar en una falta de reciprocidad en la relación muy característica de los individuos con SA.

Algunos de sus síntomas son: incapacidad en las interacciones sociales, desconocimiento de los códigos que regulan una interacción social, incapacidad para interpretar el lenguaje no verbal o limitaciones para el autocontrol. 


\subsubsection{Trastorno Bipolar tipo I (TB I)}

Según el DSM-IV-TR, el trastorno bipolar tipo I se caracteriza por un "curso clínico caracterizado por uno o más episodios maníacos o episodios mixtos". Además, añade, que "es frecuente que los sujetos también hayan presentado uno o más episodios depresivos mayores" (2002:428). Durante los episodios maníacos graves el individuo puede experimentar comportamientos violentos y provocar agresiones.

Para el diagnóstico de TB I, se establecen criterios en función del tipo del último episodio (más reciente maníaco único, hipomaníaco, maníaco, mixto, depresivo o no especificado).

Los síntomas más evidentes del TB I son: aumento de autoestima o grandiosidad, lenguaje verborreico o fuga de ideas.

\subsubsection{Trastorno Bipolar tipo II (TB II)}

EL TB II, según viene descrito en el DSM-IV-TR, se caracteriza por "la aparición de uno o más episodios depresivos mayores acompañados por al menos un episodio hipomaníaco". "Los síntomas deben provocar malestar clínicamente significativo o un deterioro social, laboral o de otras áreas importantes de la actividad del individuo" (2002:439). Es muy importante tener en cuenta que los sujetos que sufren TB II pueden no considerar como patología los episodios hipomaníacos porque, entre otras cosas, con frecuencia no recuerdan los períodos de hipomanía, por lo que el individuo sufre vacíos en la memoria de los que no siempre es consciente, lo que provoca problemas de relación con las personas de su alrededor.

Los síntomas del TB II son compartidos con los del TB I, la diferencia radica en el tipo de episodios.

\subsubsection{Trastorno Disociativo de Idendidad (TID)}

El TID se define en el DSM-IV-TR como un trastorno cuya característica esencial es la existencia de dos o más personalidades. Investigaciones confirman que lo habitual es encontrar un máximo de diez personalidades, pero se han documentado casos de hasta cien. En el TID 
"cada personalidad se vive como una historia personal, una imagen, una identidad e incluso un nombre distinto" (2002:589). Normalmente hay una personalidad primaria, con el nombre del paciente y el resto de las personalidades pueden asumir sexos distintos, vocabulario, edad, estados de ánimo, etc. En determinadas ocasiones surgen personalidades que cuentan con más presencia que las otras y pueden llegar a liderar el cuerpo del sujeto, planificando el tiempo que otorga al resto.

Algunos de sus síntomas son: huecos en la memoria, pesadillas o flashbacks característicos del trastorno de estrés postraumático (TEPT) o comportamientos suicidas.

\subsubsection{Trastorno Límite de la Personalidad (TLP)}

El DSM-IV-TR lo define como "un patrón general de inestabilidad en las relaciones interpersonales, de autoimagen y afectividad, y una notable impulsividad que comienza al principio de la edad adulta y se da en diversos contextos" (2002:790).

La sensación de abandono es constante en pacientes con TLP, sin importar si es real o imaginado y, normalmente, entienden que ese abandono implica que hay en ellos una maldad que el otro no ha sido capaz de soportar. No entienden los cambios, se enfurecen si sus planes sufren cualquier cambio imprevisto.

Inestabilidad en las relaciones personales, sensación constante de abandono, comportamientos suicidad o problemas de control de ira son algunos de los síntomas que pueden experimentar los pacientes de TLP.

\subsubsection{Trastorno Narcisista de la Personalidad (TNP)}

El TNP se basa en "un patrón general de grandiosidad, necesidad de admiración y falta de empatía que empieza al comienzo de la edad adulta y se da en diversos contextos" (DSM-IVTR, 2002:799). Es común entre los pacientes de TNP sobrevalorar sus capacidades y conocimientos e infravalorar las del resto, lo que hace que constantemente sientan que no reciben las alabanzas que creen merecer. Además, con frecuencia se dejan llevar por fantasías 
de éxito ilimitado en cualquier ámbito de su vida, se consideran brillantes, merecedores de cualquier amor que deseen.

Pueden mostrar necesidad de admiración, autoestima alta y frágil, intolerancia a las críticas, así como soberbia y arrogancia.

\subsubsection{Trastorno Obsesivo-Compulsivo (TOC)}

El Trastorno Obsesivo-Compulsivo necesita, invariablemente, de la presencia de obsesiones y compulsiones y, además, que estás sean recurrentes y lo suficientemente graves como para que la pérdida de tiempo que suponga su realización sea significativa.

- Las obsesiones se definen como “ideas, pensamientos, impulsos o imágenes de carácter persistente que el individuo considera intrusas e inapropiadas y que provocan una ansiedad o malestar significativos", a estas intrusiones se las denominan egodistónicas. El sujeto es capaz de reconocer que son producto de su mente, pero no puede evitarlas.

- Las compulsiones, por otro lado, son comportamientos o actos mentales recurrentes que el paciente realiza para aliviar el estrés que le produce las obsesiones. La realización de la compulsión no provoca en el sujeto placer, sino que las efectúa para evitar el malestar que le causa no llevarlas a cabo. Las compulsiones no están conectadas con las ideas que obsesionan (DSM-IV-TR, 2002:510-512).

Además de los comportamientos repetitivos basados en obsesiones y compulsiones, los pacientes de TOC pueden mostrar hipocondría, confinamiento para evitar situaciones de estrés y diferentes trastornos de la personalidad.

\subsubsection{Trastorno por Déficit de Atención con Hiperactividad (TDAH)}

El TDAH se define como "un patrón persistente de desatención y/o hiperactividadimpulsividad" cuyos primeros síntomas pueden aparecer antes de los 7 años (DSM-IV-TR, 2002:97). Esta desatención puede darse en situaciones académicas, laborales o sociales en las 
que el sujeto no tiende a no prestar atención a los detalles, cometer errores por descuidos o a llevar a cabo un trabajo sin reflexión previa. Además, a los pacientes de TDAH, les resulta complicado finalizar tareas, tienen una tendencia a empezar varias que van dejando sin concluir porque se distraen, lo que lleva a una desorganización que ocurre especialmente en actividades que exigen una dedicación personal y un esfuerzo mental durante un periodo de tiempo significativo.

Algunos de sus síntomas son problemas para completar actividades, dificultad para acatar normas o disforia.

\section{Resultados y conclusiones}

\subsection{Caso 1. Temperance Brennan (Bones): la perfecta asocial.}

\subsubsection{Bones: la emoción oculta.}

El caso de Temperance Brennan va a ser tomado como modelo para comparar el resto de personajes por varios motivos. En primer lugar, es la primera protagonista que aparece en el estudio, dado que no hay precedentes de féminas protagonistas que puedan ser consideradas "perfectas asociales"3 como es este caso. En segundo lugar, es importante tener en cuenta que sus problemas de sociabilización no radican en ninguna enfermedad tipificada, sino que provienen de traumas previos al punto de partida de la ficción que no han desarrollado ninguna patología ulterior. Bones, como serie, sigue la estela de House, ambas ficciones crearon a un personaje novedoso, de una sinceridad devastadora con deficiencias en las relaciones interpersonales y que han servido como referente para series posteriores.

En caso de Brennan, el trauma viene de una cadena de acontecimientos relacionada con sus padres. Cuando ella tiene 15 años cae en el sistema de adopciones al pensar que sus progenitores han fallecido en un accidente de tráfico. En su vida adulta aparece en la fícción el personaje de Max, su padre, y Bones descubre que es hija de unos ladrones de bancos. La relación con el sistema de adopciones, la historia de sus padres y sus problemas con Max en

\footnotetext{
${ }^{3}$ El término "perfecta asocial" va a recoger a personajes, en este caso femeninos, pero es independiente del género que, habiendo sufrido un suceso traumático ha desarrollado un déficit para las relaciones sociales, pero no tiene ningún trastorno de la personalidad asociado. Los TP en las series que van a ser estudiadas se utilizan como elemento definitorio, delimitando las acciones del personaje mientras que, cuando no lo tienen, gozan de ese halo abstracto que interesa al espectador, tal y como se detallaba en las primeras páginas de este artículo. Por lo tanto, el personaje no tiene la condición de "enferma" o "loca", si no de asocial.
} 
particular han hecho que Brennan sea un personaje que se mide por la desconfianza en las relaciones humanas considera que, si las evita, no pueden hacerle daño. Otro de los rasgos más acentuados de su personalidad es el hecho de que se mide por la inteligencia, abandonando las emociones por completo. El origen de esta peculiaridad se explica en el capítulo Un tiro en la oscuridad $^{4}$ en el que, mientras está en coma, tiene una ensoñación en la que habla con su madre y ambas explican las últimas palabras que tuvieron: "Y te dije que eras demasiado soñadora y emocional, tomando decisiones en base a lo que sentías. Te dije que usaras tu cerebro", a lo que Bones responde: "y nunca volví a verte”. Esta relación de sucesos traumáticos provocados consciente o inconscientemente- por los progenitores se repetirá en buena parte de los casos que van a quedar analizados.

\subsubsection{Maternidad cuestionada.}

Una de las aristas de estos personajes que más afectada se ve por el hecho de tener un trastorno de la personalidad -o ser sospechoso de padecer uno- es cómo vive la maternidad o cómo los personajes satélites juzgan la maternidad de la protagonista. Este aspecto es relevante en este caso en cuestión.

Los objetivos familiares del personaje no están, en ningún caso, estereotipados. En primer lugar, porque no desea formar una familia hasta la cuarta temporada, cuando reconoce su deseo de ser madre -influenciado por el hecho de que Ángela Montenegro va a serlo- y, en segundo lugar, porque cuando expresa su deseo afirma que quiere hacerlo en solitario, que no necesita pareja. Cuando Temperance habla de la posibilidad de ser madre en solitario es cuestionada por todos sus compañeros de trabajo. Creen que es un capricho, un reto intelectual que no va a tener implicaciones emocionales en su vida. Finalmente, cuando se convierte en madre, hecho que no está planificado, sí que cede a vivir con Booth, en cualquier caso, sus conflictos de convivencia muestran a la forense como una persona solitaria a la que le cuesta ceder su espacio por el bien de la relación. En este sentido hay que tener en cuenta que el embazo del personaje tampoco fue planeado por los guionistas, sino que la actriz, Emily Deschanel, se quedó embarazada en la vida real. Esto es importante porque, a priori, la maternidad del personaje de Temperance Brennan no es algo previsible, ni que cuadre con el resto de las

\footnotetext{
${ }^{4}$ Traducción libre de The Shot in the Dark $(8 \times 15)$.

5 Traducción libre de "And I told you you are too dreaming and emotional, making decisions on way you felt. I said you will use your brain" [...] "And I never saw you again". The shot in the dark (8x15).
} 
aristas del personaje. El tratamiento que recibió fue liviano y la maternidad sirvió como excusa a algunas tramas, pero no como línea argumental per se.

En cuanto a cómo puede afectar su problema social a su descendencia, la serie no da datos suficientes para su determinación. Sin embargo, sí que se lleva a su hija consigo cuando se convierte en prófuga -es sospechosa de un crimen que no ha cometido-, por lo que se entrevén comportamientos maternales que pudieran afectar a su hija en función de las complicaciones de su vida por consecuencias de su trabajo. No obstante, cualquier valoración al respecto no es más que una hipótesis.

\subsection{3. "Si pudiera reflejar lo que siento, lo haría".}

Las habilidades sociales pobres son una constante del personaje, la definen en su mayor parte, sin embargo, muestra impotencia ante ello en varias ocasiones. Por poner un ejemplo, en el capítulo El signo del silencio, le dice al doctor Vaziri "le aseguro que, si supiera cómo reflejar lo que siento, lo haría. Le facilitaría mucho la vida a los demás"”.

A pesar de sus dificultades para las relaciones sociales, el personaje de Temperance Brennan con cuadra con suficientes síntomas de un trastorno concreto como para considerar que lo padece. Es por esto por lo que se concluye que Temperance Brennan es un modelo de lo que se llamará "perfecta asocial", un personaje al que no se le atribuye ningún trastorno, sus deficiencias sociales vienen determinadas por un trauma durante la infancia, pero no la limita la etiqueta de "trastornada", como ocurre en otros muchos casos de protagonistas masculinos con problemas de sociabilización.

\subsection{Caso 2. Tara Gregson (United States of Tara): una ama de casa, un excombatiente y una niña asustada (entre otros)}

\subsubsection{Tara: madres y esposas.}

Tara Gregson es madre de dos hijos, está casada y padece trastorno disociativo de identidad. Cuando era pequeña sufrió abusos sexuales por parte de su hermanastro y, como no recordaba nada por el trastorno de estrés postraumático, sus padres le ocultaron lo sucedido. Fue a la

\footnotetext{
${ }^{6}$ Traducción libre de "I assure you, if I knew how to convey how I feel, I would. It seems to make other people's lives much easier". The Signs in the Silence (6×21).
} 
universidad a estudiar psicología, pero lo dejó porque sufrió una segunda violación por parte de un compañero, lo que le hizo tener varias tentativas de suicidio.

\subsubsection{Multimaternidad.}

Los objetivos familiares de Tara no están estereotipados, poco tienen que ver con casarse o formar una familia dado que ya lo hizo. No obstante, hay que destacar que se casó joven porque tuvo un embarazo no planeado de Kate. Este hecho sumando a la homosexualidad de su hijo y a la relación con su marido hace de este núcleo familiar un grupo de cuatro personajes no estereotipados. En cuanto a su maternidad, sus dos hijos fueron embarazos naturales -no adopciones, inseminación artificial, etc.- y su problema mental afecta negativamente a la vida de sus hijos en la actualidad. De hecho, Kate acaba siendo azafata de vuelo tras varios intentos de irse de casa y Marshall, que siempre ha sido el hijo fiel a su madre, termina la serie pensando en separarse de sus padres. Sus dos hijos se ven sobrepasados por la responsabilidad que conlleva para ellos cuidar de su propia madre y lidiar con todos sus álter, cada uno con un comportamiento distinto.

\subsubsection{EI TID en un guion de ficción.}

El Trastorno de Identidad Disociativo -antes Trastorno de Personalidad Múltiple- se refleja en la ficción síntoma a síntoma, pero hay algo que destaca: la aparición de cada una de las personalidades múltiples -áters- responden a una intención de guion, teniendo así una función marcada por cada personalidad.

Tara Gregson alberga en su interior, hasta 24 personalidades, aquellas que cobran protagonismo son:

- Alice: conservadora, hogareña, servicial, controladora. Inspirada en Mimi, una mujer que dirigía la casa de acogida en la que estuvo Tara, pero que no recuerda.

- Función: aparece cuando la personalidad principal, Tara, no puede mantener el control sobre algo.

- T: álter adolescente, con comportamientos hipersexualizados.

- Función: aparece cuando Tara no es capaz de afrontar algo. T no trata temas emocionales, simplemente los ignora con fiesta y alcohol. Además, es impulsiva y sus relaciones son muy inestables, dos síntomas característicos de las personas 
con TID. La inestabilidad en sus relaciones se debe a que sus acciones afectan constantemente a Tara: infidelidades a su marido, insultos a su hermana, etc.

- Buck: es un álter masculino, agresivo y machista.

- Función: cuando Tara no quiere mantener una conversación, Buck aparece para cortarla de raíz sin ofrecer explicaciones. Este alter supone el cumplimiento del síntoma de personalidades agresivas.

En las personas con TID hay un síntoma muy llamativo que ocurre con frecuencia y se basa en que una de las personalidades administra el tiempo de las otras para que, en cierta medida, cada áter pueda disponer de tiempo de protagonismo. Reflejar esto en la serie fue una de las ideas más interesantes de sus tres temporadas. En el capítulo El dedo entero ${ }^{7}$, cuando Tara, para recuperar el control, organiza una reunión de alteras que ella llama "United States of Tara", para organizar qué tiempo cede a cada personalidad. Esta reunión supone a la vez, la entrada en un estado de coconsciencia ${ }^{8}$ o conciencia dividida ya mostrado con anterioridad. Este estado supone que el huésped y la personalidad principal comparten una misma experiencia.

\subsection{Caso 3. Carrie Mathison (Homeland): Agente bipolar y madre.}

\subsubsection{Carrie contra todos.}

La construcción del background de la agente de la CIA se basa, como en los otros casos, en las relaciones paternofiliales. Frankie, su padre, es bipolar y ha criado a sus dos hijas bajo la concepción de que su madre les abandonó por su enfermedad. Es por esto que el personaje de Carrie Mathison oculta su trastorno bipolar, no mantiene relaciones interpersonales más que con su hermana y vive con la creencia de que, de entregarse a alguien, acabaría siendo abandonada por el TB.

\subsubsection{Mamá se va a la guerra.}

Se queda embarazada en el episodio Sigue dando positivo ${ }^{9}$ del sargento Brody cuando lo creía sospechoso de estar preparando un atentado terrorista en Estados Unidos -conjeturas que finalmente se confirman-. Cuando Brody muere asesinado, ella abandona el país y a su hija

\footnotetext{
${ }^{7}$ The Full Fuck You Finger (3x03).

${ }^{8}$ Traducción libre de coconcious.

${ }^{9}$ Still Positive (3x06).
} 
recién nacida para coger un cargo de mayor rango, a partir de entonces, su hermana es la encargada de cuidar de su hija.

Carrie no tiene objetivos familiares estereotipados, de hecho, no decide ser madre, se queda embarazada sin planificarlo y, cuando obtiene un cargo importante en Pakistán no duda en dejar a su hija con su hermana. En el capítulo Se veía venir hace tiempo ${ }^{10}$, Maggie ha de recordarle a Carrie que su madre abandonó a su marido y sus dos hijas igual que hizo la propia Mathison cuando dejó a su padre enfermo y a su hija a cargo de su hermana. A lo largo de sus idas y venidas de Pakistán en la temporada cuatro, uno de los conflictos a los que se tiene que enfrentar Carrie es que no sabe cuidar de su hija, no tiene instinto maternal y que ésta es un recuerdo vivo de Brody.

\subsubsection{Bipolaridad intermitente.}

Carrie Mathison es una bipolar de manual. La confirmación del diagnóstico la se encuentra en el capítulo El Chaleco $^{11}$ en el que, tras una explosión, la agente de la CÍA entra en un episodio maníaco tal y como confiesa Maggie, su hermana, a Saúl, su mentor. En el citado capítulo, se justifican casi todos los síntomas presentes en el TB tipo I, algunos de ellos son:

- Grandiosidad. Carrie le confiesa a Saul que sufre TB desde la facultad, cuando escribió un manifiesto asegurando que había reinventado la música.

- Lenguaje verborreico, fuga de ideas, distraibilidad y agitación psicomotora presentes a lo largo de todo el capítulo. Sin embargo, es capaz, en pleno episodio maníaco de establecer un código de colores para aplicar a todas las pruebas, con ideas aparentemente inconexas, para avanzar en el caso. Compilación que descifra Saúl. Esto se debe a la necesidad de que este tipo de personajes se muestren útiles y funcionales a pesar de su trastorno.

Así pues, en base al cumplimiento del cuadro sintomático de Carrie Mathison, puede afirmarse, sin lugar a duda, que la documentación de la ficción es correcta. No obstante, la utilización del trastorno de Carrie acaece en tanto es necesario para la trama que la protagonista entre en un episodio maníaco, pero, al margen de esos momentos, el tratamiento que recibe la enfermedad

\footnotetext{
${ }^{10}$ Long Time Coming (4x12).
}

${ }^{11}$ The Vest $(1 \times 11)$. 
es mínimo, por lo que no se muestra la verdadera influencia que recibiría un paciente con TB en su funcionalidad como agente de la CIA.

\subsection{Caso 4. Hanna Horvath (Girls). Una millennial en Manhattan.}

\subsubsection{El abandono.}

La serie parte con los padres de Hannah dándole la noticia de que no van a seguir manteniendo su vida en Nueva York, así pues, ha de conseguir un trabajo para sobrevivir. Sin embargo, una vez vista hasta su quinta temporada, la complejidad psicológica del personaje no está delimitada por un hecho traumático. Hannah ha vivido con sus padres y, a pesar de que tiene mejor relación con su padre -muestra más amabilidad para con su hija-, su personalidad tiene los mismos pilares que la de su madre, quien se caracteriza por su egocentrismo y dramatismo ante cualquier hecho cotidiano.

De su pasado apenas se sabe que ha sufrido bullying por su sobrepeso, así como que tuvo TOC -Trastorno Obsesivo Compulsivo- cuando iba al instituto, enfermedad que se recupera para los capítulos Recaída, A gatas y Juntos $^{12}$, donde Hannah pierde el control ante su ruptura con Adam, su mala relación con sus padres y su bloqueo creativo con el libro que está escribiendo. No obstante, el desarrollo de la enfermedad dura los tres capítulos mencionados y no vuelve a repercutir en el presente del personaje. Por lo demás, Hannah se caracteriza por considerar a sus amigas su familia, una mentalidad que casa perfectamente con la generación millennial a la que representa.

\subsubsection{La no maternidad.}

Girls, al centrarse en el retrato de la generación millennial, hace que Hannah, una chica de 23 años no tenga objetivos familiares estereotipados ni relacionados con la maternidad en ninguna de sus relaciones. A lo largo de las cinco primeras temporadas se nos presenta una transformación basada en el camino hacia la madurez, es por eso que la protagonista vive la mayor parte de la serie con una actitud ante la vida que queda muy lejos de plantearse futuro a largo plazo.

${ }^{12}$ It's Back (2x08), On All Fours (2x09) y Together (2x10). 


\subsubsection{TOC fugaz.}

El TOC de Hannah Horvath se muestra en los ya citados episodios Recaída, A gatas y Juntos, por lo que, a pesar de que se afirme que lo tiene desde el instituto, no se puede tener en consideración como un factor determinante en la construcción del personaje al margen de en los episodios citados. No obstante, se reflejan síntomas característicos de la enfermedad como que las obsesiones y compulsiones que supongan una pérdida de tiempo significativa. Acompañando a las conductas apremiantes, el confinamiento para evitar situaciones de estrés y el trastorno de ansiedad, también se muestran de forma exclusiva en el periodo en el que se explora el TOC. Sin embargo, las preocupaciones hipocondríacas que se encuentran en los pacientes obsesivo-compulsivos son la única característica constante en su construcción y, de hecho, esta forma de comportamiento es compartida con su madre, por lo que está muy arraigada en el personaje.

Cuando Hannah siente que está perdiendo el control tras su ruptura con Adam, sumado al estrés que le provoca haberse gastado el adelanto de un libro que no se siente capaz de escribir, sufre una complicación de un trastorno que tenía controlado con medicación y visitas al psicólogo.

Respecto a este carácter fugaz, el DSM-IV-TR, aclara que experiencias complicadas pueden llevar a una intensificación de las compulsiones pero que, rara vez, la aparición de estos rituales es aguda -como ocurre en Hannah-, lo habitual es que sea gradual. Además, tampoco es común que los síntomas desaparezcan por completo, por lo que tanto la aparición como la posterior ausencia del TOC en apenas tres episodios resulta brusca y poco plausible.

\subsection{Caso 5. Sarah Manning (Orphan Black): el concepto de familia.}

\subsubsection{La hija de la abuela.}

Aunque el objeto de estudio de la muestra es el personaje de Sarah Manning por su condición de protagonista, la ficción muestra características que hay que poner de relieve. Para empezar, es una serie coral, aunque tanto en la focalización como en las tramas, el peso de Sarah sea ligeramente superior. En segundo lugar, está la posible lectura de que todas las clones son partes de una misma persona, en tanto que cada una tiene resaltadas características de la personalidad muy diferentes y, en conjunto, son más que la suma de sus partes, podría verse pues, a todos los clones como un completo y complejo personaje. Si bien no es la consideración 
que va a predominar en el presente análisis que va a centrarse en Sarah, era importante al menos mencionarla. Por último, hay varios personajes que cabrían perfectamente en la muestra pero que han sido excluidos por no ser protagonistas, a saber: Alison Hendrix, Helena y Rachel Duncan, tres personajes evidentemente afectados por trastornos de la personalidad desencadenados, entre otros factores, por los núcleos familiares y/o experiencias traumáticas.

La concepción familiar de Orphan Black, a nivel general, se basa en los conceptos de familia biológica versus familia adoptiva, en el papel del progenitor -habitualmente madre-, en las familias monoparentales o en la conformación del sujeto en condiciones de ausencia total de padres. Sarah es adoptada, ha sido criada por la Señora S, un personaje construido en base a dos conceptos: maternidad e independencia. Es protectora, la cuidadora de Sarah y Félix, ambos adoptados. Biológicamente, Sarah es 'hija' -clonada a partir de su carga genética- de Kendall Malone, madre de la Señora S, es decir, Sarah ha sido creada a partir de la madre de la madre. No es casualidad que, de todos los clones, ella sea la única que puede tener hijos, por esta razón Sarah, además, es madre de Kira.

Sarah es un personaje que se caracteriza por la desconfianza, tanto de lo que conoce como de lo que no, es por esto que sus relaciones son inestables. Es un personaje impulsivo, pasional, que hace y después piensa en las consecuencias.

\subsubsection{Sarah, la madre.}

La maternidad es tan importante en Orphan Black, que necesariamente ha sido tratada en la introducción general, a pesar de ello, a modo resumen, cabe destacar que la maternidad del personaje es única entre sus iguales, y eso es lo que la hace especial dentro de la ficción y le da la condición de protagonista en una serie coral.

En cuanto a la forma de ejercer su maternidad, desde los inicios de la serie, la señora $\mathrm{S}$ se encarga de marcar el fracaso de Sarah con Kira. La inestabilidad de Sarah hace que sea un personaje que desaparece durante meses y, cuando aparece, va en busca de su hija. En el arranque de la serie su intención es vender droga que le ha robado a su expareja para poder irse con Kira de la ciudad. No obstante, a lo largo de las cinco temporadas, una de las cosas que Sarah tiene que aprender es a ser madre, a proteger a Kira como la señora S las ha protegido a ellas y a Félix. 


\subsubsection{Sarah Manning: sobreviviendo a la familia.}

Al igual que ocurre con el personaje de Temperance Brennan (Bones), Sarah Manning no cumple con el cuadro diagnóstico de ninguno de los trastornos de la personalidad aquí estudiados -SA, TB I o II, TID, TLP, TNP, TOC o TDAH-.

A pesar de los orígenes familiares de Sarah y de la posibilidad de traumas existentes, se configura como una luchadora, independiente y empoderada en un entorno difícil. Es hija, madre y hermana y sus características principales son el instinto de protección y la desconfianza ante lo externo. Así pues, Sarah, siguiendo los pasos de Brennan ocho años después, se convierte en una versión de mayor complejidad psicológica y con tramas mejor exploradas de la "perfecta asocial", un personaje cuya representación en estas series es tan valiosa como difícil de encontrar.

\subsection{Caso 6. Sonya Cross (The Bridge): ¿asperger?}

\subsubsection{Verdad sin edulcorar.}

La construcción de este personaje es muy similar a la construcción de Carrie Mathison (Homeland). Ambas ostentan un cargo relacionado con la justicia, Sonya es policía de El Paso y Mathison agente de la CIA, ambas están rodeadas de personajes masculinos, las dos tienen un mentor que supone un personaje de más edad y con mayor autoridad-en el caso de Sonya es Hank y en el de Carrie es Saúl- y ambas están intermitentemente afectadas por un problema de sociabilización. Con respecto a esto último, Mathison era abiertamente bipolar, en el caso que nos atañe, Sonya presenta síntomas de asperger.

La vida familiar de Sonya está marcada por el asesinato de su hermana y la drogadicción de su madre. Cuando la hermana se Sonya es asesinada, ella tiene 15 años y se queda con Hank, quien la impulsa a ser policía. Es interesante la contradicción que presenta la relación entre estos dos personajes: Sonya se caracteriza por llevar la verdad como filosofía de vida, no sabe mentir. Hank, por su parte, le ha ocultado a Sonya que fue él quien disparó al asesino de su hermana -en un gesto de vehemencia-, lo que provocó que el criminal quedara vivo, pero sin poder articular palabra y sin apenas enterarse de lo que ocurre a su alrededor, dejando a Sonya 
sin respuestas para siempre. No puede escaparse que su mentor es un personaje masculino y el progenitor que ha estado siempre ausente ha sido su padre.

\subsubsection{Sin familia, sin hijos.}

Sonya no plantea objetivos familiares estereotipados basados en modelos tradicionales como ser madre, casarse, etc. Su personalidad está tan alejada de las relaciones sociales convencionales que ni se plantea la posibilidad, ni el espectador lo espera.

\subsubsection{Sonya Cross: diagnosticando a una agente de policía.}

Sonya Cross presenta todos los síntomas del SA. En primer lugar, y de manera constante, la incapacidad en las relaciones sociales; en segundo lugar, Sonya está obsesionada por las normas y la sinceridad que aplica cada día en el trabajo en el que vive absorta -conducta, intereses y actividades repetitivos y restringidos son otros de los síntomas del SA-. En el episodio Calaca $^{13}$, su compañero Marco recibe una llamada de su esposa y Sonya no entiende que la llamada ocurra sin motivo aparente, no entiende las normas de conversación. En el episodio La bestia ${ }^{14}$, ocurre un hecho que deja ver su incapacidad para interpretar el lenguaje no verbal. Marco ha tenido una relación extramatrimonial y se ha dejado olvidada la cartera en casa de la chica, la billetera acaba en manos de Sonya. Ella, en un gesto inocente, decide devolvérsela a su compañero delante de su mujer y sus hijos, pero en ningún momento se percata de que es una situación tensa y que su esposa sospecha lo que está ocurriendo.

$\mathrm{Su}$ obsesión por los detalles, que la hace única en su trabajo, lleva a su equipo a la resolución del misterio sobre el lugar donde está secuestrado el hijo de Marco. No obstante, si hay algo que llama la atención es que recuerda, días después de haber estado en la casa del sospechoso, el sonido de una tubería que no debería estar ahí, de lo que se extrae, que tiene una excelente memoria auditiva característica de una buena parte de los pacientes de asperger.

${ }^{13}$ Calaca $(1 \times 02)$.

${ }^{14}$ The Beast $(1 \times 05)$. 


\subsection{Caso 7. Rachel Goldberg (Unreal): Cuatro enfermedades y una madre trastornada.}

\subsubsection{Productora y manipuladora a partes iguales.}

Probablemente Rachel sea el personaje más complejo y completo del presente estudio. Manipuladora, mentirosa, tergiversadora y cruel, son las características que la hacen la mejor en su trabajo, Rachel es productora de un reality en el que un chico elige entre varias chicas para encontrar el amor. Sin embargo, cuando se sincera, deja entre ver buenas intenciones, es feminista, quiere escribir un libro, ayudar a la gente y odia la telebasura para la que trabaja. La estabilidad para Rachel es un objeto de deseo inalcanzable por su personalidad, ya que vive y se maneja mejor en el caos.

Se define porque le asusta la felicidad, tal y como sentencia en el capítulo Verdad ${ }^{15}$, tiene problemas de alcohol y autocontrol, de hecho, siempre parece que está a punto de explotar sentimentalmente hablando, pero es capaz de dejarse llevar por amor, tal y como ocurre en el final de la primera temporada, cuando finalmente es humillada por sus sentimientos.

Su madre es psiquiatra e investigadora, una mujer entregada a su trabajo que trata clínicamente tanto a su marido como a su hija. Al primero, le diagnostica Trastorno Bipolar (TB) y lo medica para ello; a Rachel le ha llegado a asociar hasta cuatro trastornos mentales: Hiperactividad (TDAH), Trastorno Límite (TLP), Narcisista (TNP) y TB, con sus correspondientes medicamentos y terapias ofrecidas por ella misma. La razón de este hipercontrol sobre los tratamientos de su hija se debe a que a los doce años Rachel fue violada por un paciente de su madre y ésta, para salvaguardar su investigación, ocultó lo ocurrido y comenzó medicación y sesiones de terapia. Tal y como ella misma asegura, las normas médicas éticas de no tratar a familiares y amigos son arbitrarias, lo que evidencia un grave problema con las normas, conflicto que también afecta a Rachel.

En un punto anterior al inicio de la serie, Rachel estuvo ingresada en un centro psiquiátrico, experiencia que repetirá al final de la segunda temporada, cuando pierda de nuevo el control definitivamente. Además, sus problemas con las normas la llevaron a prisión, también en un momento previo al punto de partida de la ficción, de hecho, en el pistoletazo de salida, Rachel está en libertad condicional gracias a Quinn, su jefa, que quiere tenerla a su servicio dado su utilidad dentro del programa.

${ }^{15}$ Truth (1x05). 


\subsubsection{La maternidad inconcebible.}

Rachel no es madre, ni muestra en la serie deseos de serlo. Su inestabilidad psíquica y emocional hace que su mayor preocupación sea cuidar de ella misma cuando pierde el control.

\subsubsection{Cuadro psicológico complejo.}

El diagnóstico psicológico de Rachel es el que presenta mayor complejidad de entre los sujetos de estudio. Esto se debe principalmente a que su madre le acusa hasta cuatro trastornos de la personalidad distintos, tal y como Rachel le increpa en el capítulo Madre ${ }^{16}$. Estas enfermedades son:
A. Trastorno bipolar (TB).
B. Trastorno Límite de la Personalidad (TLP).
C. Trastorno Narcisista de la Personalidad (TNP).
D. Trastorno por Déficit de Atención con Hiperactividad (TDAH).

A pesar de los cuatro trastornos diagnosticados por su propia madre, lo más interesante de Rachel es que sufre síntomas de todos, pero no cuadra al completo con ninguno, ni la serie se posiciona sobre si Goldberg es o no una trastornada.

El TB, si bien cumple alguno de los síntomas como el lenguaje verborreico o la implicación excesiva en actividades placenteras con un alto potencial para producir consecuencias graves, el componente más importante para haberlo tenido en cuenta es que su padre lo sufre -al menos según la opinión de su madre-. No obstante, no es el TP que mejor cuadra con el personaje. Por otro lado, el TLP, se asocia a los abusos sexuales en la infancia, por lo que cuadra con el trauma de Rachel, pero no con el cuadro sintomático en sí.

Por último, los trastornos NP y DAH, sí que cuadran con los supuestos síntomas de Rachel, sin embargo, ni ella ni ciertas personas de su alrededor reconocen a Rachel como una trastornada si no simplemente como una persona con problemas de inestabilidad emocional. Es por esto que no se puede determinar si verdaderamente sufre algún TP, hasta que la serie -aún en emisiónse posicione, si es que llega a hacerlo.

${ }^{16}$ Mother (1x03). 


\subsection{Caso 8. Maria Bamford (Lady Dynamite): ¿cómo se vive con trastorno bipolar?}

\subsubsection{EI TB en pantalla.}

María Bamford, personaje y actriz, crea la serie Lady Dynamite para incorporar capítulos reales de su enfermedad, Trastorno Bipolar tipo II (TB II), y así romper tabúes y estigmas que se asocian al TB II. La idea es hacer llegar al gran público, en un tono divertido y surrealista, a qué se enfrentan cada día las personas que sufren la enfermedad: huecos en la memoria, inestabilidad en sus parejas, problemas para encontrar trabajo, etc.

\subsubsection{Ni soy madre, ni quiero serlo.}

A lo largo de la primera temporada de la serie, María confiesa en varias ocasiones que no le gustan los niños, las interacciones derivadas de sus relaciones con éstos le suponen un elemento estresante.

\subsection{Rompiendo estereotipos.}

Una de las principales diferencias de María Bamford con respecto al resto de sujetos de estudio es el objetivo para el que se crea el personaje. Es por esto que características que se asocian a otros sujetos, como la funcionalidad en su trabajo, no afectan a este personaje, no hay una necesidad imperante de hacerla plenamente válida, sino al contrario, su escasa funcionalidad es un requisito para los objetivos de la serie: mostrar el trastorno desde dentro.

De nuevo, al igual que ocurre con Carrie Mathison, el personaje cumple con todos los síntomas asociados a su trastorno, de hecho, en varias ocasiones, Bamford, afirma que es hipomaníaca, un tipo de episodio que caracteriza a su trastorno. En cuanto a la documentación de la enfermedad, hay poco que añadir, dado que la serie es una visión personal de cómo afecta el TB II desde una paciente del trastorno. No obstante, cabe destacar que amistades, interacciones sociales de otro tipo -familiares, de pareja y laborales- se ven afectadas frecuentemente por el trastorno de María y este es el punto central sobre el que gira la serie.

\subsection{Conclusiones: desmontando a las locas.}

Han quedado analizados los ocho personajes que conforman el corpus en tres aspectos relevantes en su construcción: a) descripción del personaje en base a su pasado, b) maternidad, y c) trastorno de la personalidad reflejado en la serie. 
Así, podemos concluir que hay tres tipos de maternidad:

- Maternidad cuestionada: Temperance Brennan (Bones), Tara Gregson (United States of Tara).

- Malas madres: Carrie Mathison (Homeland) y Sarah Maning (Orphan Black) abandonan a sus hijos.

- No madres: Hannah Horvath (Girls), por desinterés; el resto Sonya Cross (The Bridge), Rachel Goldberg (Unreal) y Maria Bamford (Lady Dynamite) por inestabilidad y/o TP asociado.

Además, en cuanto a los límites que el TP establece a los personajes, se encuentran:

- Perfectas asociales: personajes que tienen un background traumático, han crecido con problemas y han desarrollado dificultades para establecer relaciones interpersonales, pero no vienen delimitadas por un trastorno de la personalidad.

- Casos: Temperance Brennan (Bones), Sarah Maning (Orphan Black) y Rachel Goldberg (Unreal) ${ }^{17}$.

- Trastornadas: se incluyen aquí personajes con un TP asociado que las delimita.

- Delimitadas en general (trabajo, relaciones personales, hijos, etc). Casos de Tara (United States of Tara), Carrie Mathison (Homeland), Hannah Horvath (Girls) ${ }^{18}$ y Maria Bamford (Lady Dynamite).

- Delimitadas en lo personal no en el trabajo: Sonya Cross (The Bridge).

Así pues, se concluye, en primer lugar, que hay pocos personajes femeninos con problemas de relaciones sociales que gocen de la ambigüedad que los hace aún más interesantes para el espectador. En segundo lugar, estas féminas, en su mayoría, vienen delimitadas por síntomas de algún trastorno de la personalidad que hacen que sus acciones sean cuestionadas, que las

\footnotetext{
${ }^{17}$ Rachel Goldberg se ha incluido en este grupo por dos motivos. En primer lugar, porque en la serie no se determina si tiene o no TP, lo que a nivel actancial hace de ella un personaje sin límites, que pierde el control por inestabilidad emocional y porque, aunque tenga la etiqueta de trastornada, en determinadas ocasiones, el personaje se empodera y se reivindica como libre, ingobernable y con una capacidad apabullante para su desarrollo laboral. En segundo lugar, se incluye en este grupo porque, de padecer algún TP, pesa sobre ella la duda de cuatro opciones -de momento-, ni siquiera su madre, prestigiosa psiquiatra investigadora, es capaz de determinar qué le ocurre ni si le ocurre algo verdaderamente, lo que hace de Rachel Goldberg un personaje con la ambigüedad característica de Temperance Brennan o Sarah Maning.

${ }^{18}$ Se tienen en cuenta los tres episodios analizados en los que Hannah desarrolla TOC, no el total de la serie.
} 
debilita como personaje en determinados momentos de la trama y necesitan de la ayuda de algún que otro compañero -normalmente masculino- para poder cumplir su objetivo.

Es difícil encontrar a personajes femeninos en la ficción estadounidense actual que cuenten con un blackground traumático, problemas de relaciones sociales y que gocen de la independencia de Brennan, Maning o Goldberg. Lo habitual, en estos casos que de por sí son reducidos, es encontrar a locas, trastornadas y personajes que necesitan ayuda no por las dificultades que entrañe su trama, si no por su inestabilidad psicológica.

\section{Referencias Bibliográficas}

Argyle, M. (1978). Psicología del comportamiento interpersonal. Madrid: Alianza Editorial, S.A.

Arias, F. (1997). El proyecto de investigación. Caracas: Episteme.

De la Torre, T. (2016). Historia de las series de televisión. Barcelona: Roca Editorial.

De la Torre, T. (coord.) (2016). La medicina en las series de televisión. Barcelona: Fundación Dr. Antonio Esteve.

Guarinos, V. (2008). ¿Representar o simular? Esa es la pregunta: los límites de la representación. Icono14, 6 (10), 1-18.

Han, B. (2010). La sociedad del cansancio. Barcelona: Herder.

Martos, J., Ayuda, R., González, A., Freire, S. y Llorente, M. (2012). El síndrome de Asperger. Evaluación y tratamiento. Madrid: Síntesis.

Millon, T. (coord.) (2006). Trastornos de la personalidad en la vida moderna. Barcelona: Elsevier Masson.

Pacheco, L. (2015). Héroes asociales en la ficción televisiva estadounidense actual: Patologías clínicas y psicoanalíticas de los personajes de House a Mr. Robot (inédito).

VVAA. (2002). DSM-IV-TR. Manual diagnóstico y estadístico de los trastornos mentales. Barcelona: Elsevier Masson. 


\section{Referencias videográficas}

Araki, T. (Director y productor). (2006-2007). Death Note. [Serie de televisión]. Japón: D.N. Dream Patners.

Brady, P. y Hurwitz, M. (Productores) \& Cohen, R. (Director). (2016-Actualidad). Lady Dynamite. [Serie de televisión]. Estados Unidos: Netflix.

Caballero, M.J. y De Leyva, C. (Productoras) \& Freixas, P. (Director). (2009-2014). Los Misterios de Laura. [Serie de televisión]. España: TVE.

Cody, D. (Productor) \& Zisk, C (Director). (2009-2011). United States of Tara. [Serie de televisión]. Estados Unidos: Showtime.

- Jones, R. (Guionista) \& Zisk, C. (Director). (2011). The Full Fuck You Finger. [Episodio serie de televisión]. Estados Unidos: Showtime.

Davids, J. (Productor) \& Kershaw, G. (Director). (2005-Actualidad). Criminal Minds. [Serie de televisión]. Estados Unidos: CBS.

Dunham, L. (Productora y directora). (2012-Actualidad). Girls. [Serie de televisión]. Estados Unidos: HBO.

- Dunham, L., Rubinshteyn, S. y Schoeneman, D. (Guionistas) \& Peretz, J. (Director). (2013). It’s Back. [Episodio serie de televisión]. Estados Unidos: HBO.

- Dunham, L. y Konner, J. (Guionistas) \& Dunham, L. (Directora). (2013). On All Fours. [Episodio serie de televisión]. Estados Unidos: HBO.

- Apatow, J. y Dunham, L. (Guionistas) \& Dunham, L. (Directora). (2013). Together. [Episodio serie de televisión]. Estados Unidos: HBO.

Fawcett, J. (Productor y director). (2013-Actualidad). Orphan Black. [Serie de televisión]. Canadá-EEUU: BBC.

Frost, M. y Lynch, D. (Productores) \& Lynch, D. (Director). (1990-1991). Twin Peaks. [Serie de televisión]. Estados Unidos: ABC.

Fuller, B. (Productor) \& Rymer, M. (Director). (2013-2015). Hannibal. [Serie de televisión]. Estados Unidos: NBC.

Gordon, H. (Productor) \& Linka, L. (Directora). (2011-Actualidad). Homeland. [Serie de televisión]. Estados Unidos: Showtime.

- Stiehm, M. y Johannessen, C. (Guionistas) \& Johnson, C. (Director). (2011). The Vest. [Episodio serie de televisión]. Estados Unidos: Showtime. 
- Cary, A. y Hall, B. (Guionistas) \& Linka, L. (Directora). (2013). Still Positive. [Episodio serie de televisión]. Estados Unidos: Showtime.

- Stiehm, M. (Guionista) \& Linka, L. (Directora). (2014). Long Time Coming. [Episodio serie de televisión]. Estados Unidos: Showtime.

Hanson, H. (Productor) \& Toynton, I. (Director). (2005-2017). Bones. [Serie de televisión]. Estados Unidos: Fox.

- Lin, J. y Nathan, S. (Guionistas) \& Little, D.H. (Director). (2011). The Signs in the Silence. [Episodio serie de televisión]. Estados Unidos: Fox.

Heller, B. (Productor) \& Long, C. (Director). (2008-2015). The Mentalist. [Serie de televisión]. Estados Unidos: CBS.

Manos, J. (Productor) \& Dahl, J. (Director). (2006-2013). Dexter. [Serie de televisión]. Estados Unidos: Showtime.

Marlowe, A.W. (Productor) \& Bowman, R. (Director). (2009-2016). Castle. [Serie de televisión]. Estados Unidos: ABC.

McDormand, F. y Hanks, T. (Productores) \& Cholodenko, L. (Directora). (2014). Olive Kitteridge. [Serie de televisión]. Estados Unidos: HBO.

Misiano, V. (Productor) \& Schultz, M. (Director). (2014-Actualidad). The Mysteries of Laura. [Serie de televisión]. Estados Unidos: NBC.

Pizzolato, N. (Productor) \& Fukunaga, C.J. (Director). (2014-2015). True Detective. [Serie de televisión]. Estados Unidos: HBO.

Shapiro, S.G. y Noxon, M. (Productores) \& O'Fallon, P. (Director). (2015-Actualidad). Unreal. [Serie de televisión]. Estados Unidos: Lifetime.

- Weinstein, D. (Guionista) \& Briesewitz, U. (Directora). (2015). Mother. [Episodio serie de televisión]. Estados Unidos: Lifetime.

- Shapiro, S.G. (Guionista) \& Werner, P. (Director). (2015). Truth. [Episodio serie de televisión]. Estados Unidos: Lifetime.

Shore, D. (Productor) \& Yaitanes, G. (Director). (2004-2012). House. [Serie de televisión]. Estados Unidos: Fox.

Smail, S. (Productor y director). (2015-Actualidad). Mr. Robot. [Serie de televisión]. Estados Unidos: USA Network.

Stiehm, M. y Reid, E. (Productores) \& Dahl, J. (Director). (2013-Actualidad). The Bridge. [Serie de televisión]. Estados Unidos: Fx. 
- Reid, E. (Guionista) \& Mimica-Gezzan, S. (Director). (2013). Calaca. [Episodio serie de televisión]. Estados Unidos: Fx.

- Spalding, E. (Guionista) \& Holder-Payton, G. (Director). (2013). The Beast. [Episodio serie de televisión]. Estados Unidos: Fx.

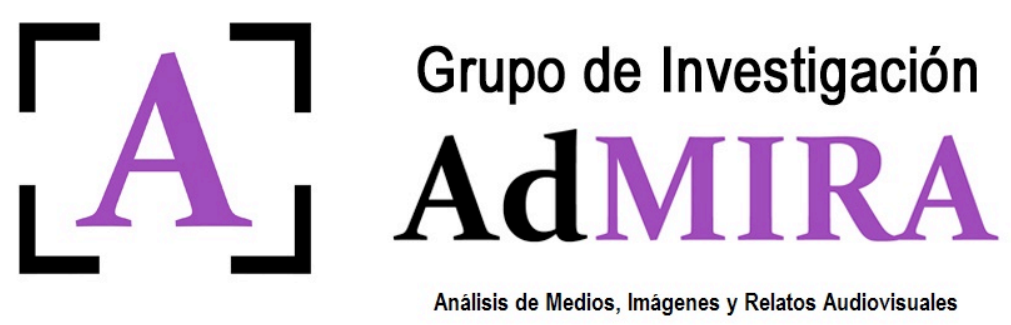

